\title{
Fast many-core solvers for the Eikonal equations in cardiovascular simulations
}

\author{
Daniel Ganellari \\ Karl Franzens University of Graz \\ Institute for Mathematics and Scientific Computing \\ Graz, Austria \\ Email: daniel.ganellari@edu.uni-graz.at
}

\author{
Gundolf Haase \\ Karl Franzens University of Graz \\ Institute for Mathematics and Scientific Computing \\ Graz, Austria \\ Email: gundolf.haase@uni-graz.at
}

\begin{abstract}
Simulation of one heart beat which faithfully account for biophysical details involved in cardiac electrophysiology and mechanics are still far away from real time performance, even when employing several thousands of compute nodes. Therefore, a simpler model based on the Eikonal equation will be considered. This model could be of great utility as a tool for generating activation and repolarisation sequences and its concomitant electrocardiogram by replacing the PDE part of the bi-domain equations with the Eikonal equations, while retaining the ODE parts to account for the full mechanistic detail relevant to ECG computation. Further, the approach can be extended to use Eikonal-based activation sequences as a driver for mechanical contraction models. We will address the implementation of an Eikonal solver for Shared Memory (OpenMP) with a low memory footprint. This solver will be transferred for a coarse model onto a tablet computer and other handheld devices for clinical use.

Due to the splitting of the wave front (described by the Eikonal equations), the parallel version results in a slightly different convergence history and in minor differences in the solution. A future CUDA implementation of the parallel algorithm will reduce the run time further such that also interactive simulations will be possible.

Index Terms-Hamilton-Jacobi equation, Eikonal equation, tetrahedral mesh, parallel algorithm, shared memory multiple-processor computer system, CUDA, GPU, Android
\end{abstract}

\section{INTRODUCTION}

There is a broad range of applications onto which the solutions to Eikonal equations apply. These applications vary from computer vision, image processing to geoscience, medical imaging and cardiac electrophysiology. The Eikonal equation is encountered mostly in problems of wave propagation and in problems of finding the shortest paths, possibly with inhomogeneous or anisotropic metrics due to material properties. In cardiac electrophysiology [1], action potentials on the heart can be represented as moving interfaces that can be modeled with certain forms for the Eikonal equation. As described in [2], many of these cases present a clear need to solve such problems on fully unstructured meshes. In cardiac simulations for example, the domain can be accurately and efficiently

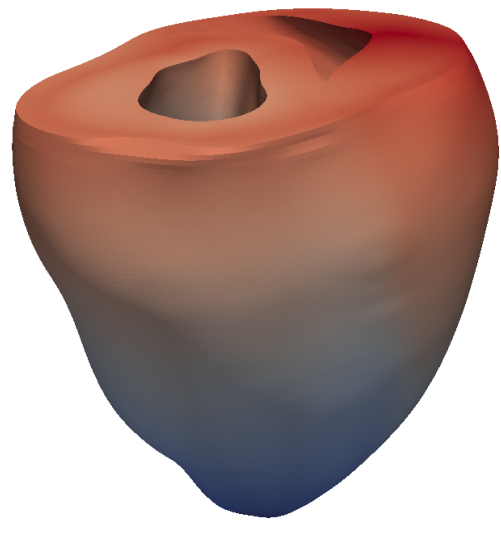

Fig. 1. Heart geometry used for the computations. Arrival time $\varphi(x)$ ranging from 0 (bottom) to 1 (top).

captured by using triangle meshing strategies for surfaces combined with tetrahedral meshing of the interior, see Figure 1. To provide solutions on such unstructured threedimensional meshes it is needed to build efficient and fast algorithms.

Recent work in [3] has shown that building an efficient 3D tetrahedral Eikonal solver for multicore and SIMD architectures poses many challenges.

First the algorithm should be independent on the order of updates and pose no dependencies between the concurrent updates which is key in order to have an easily parallelizable algorithm. Second, especially in the SIMD architecture where the available memory and registers are limited, it is important to keep the memory footprint low in order to achieve a good throughput and computational density. Third, the vertex valences of the 3D unstructured meshes can be both quite high and can be highly variable across the mesh, posing additional challenges to SIMD efficiency as described in [3]. Our SIMD implementation uses a different strategy to deal with this issue as explained in section $\mathrm{V}$, which totally avoids the uneven workload for the threads, improves the coalesced memory access and 
achieves a better throughput.

In our work we develop a framework similar to the previously proposed FIM methodology [3], [4], [5] for tetrahedral meshes, but with many differences especially in the algorithmic structure for streaming architectures. We improved the algorithm, implemented it on various hardware with very good results and also transferred the solver for a coarse model to a tablet computer and other hand-held devices for potential clinical use. To give the users a better graphical experience we will realize an interactive change of material properties on the tablet computer and also give them the possibility to recompute the solution directly using the recent changes. The final goal is to be able to perform an interactive pre-surgery planning by using our application. This paper will also provide algorithmic and implementation details followed by comparative results for the shared memory and CUDA implementation. We have implemented the local solver for android devices which has shown satisfactory results related to the OpenMP parallelization on ARM processors and great results related to the CUDA implementation on Android. The performance was improved by a factor of 6 compared to OpenMP implementation on Android.

\section{MATHEMATICAL DESCRIPTION OF THE EIKONAL EQUATION.}

The Eikonal equation is a special case of the HamiltonJacobi partial differential equations (PDEs), encountered in problems of wave propagation.

This Eikonal equation is a non-linear PDE

$$
\begin{cases}|\nabla u(x)|=F(x), & x \in \Omega \\ u=0, & x \in \partial \Omega\end{cases}
$$

where $\Omega$ is an open set in $\mathbb{R}^{n}$ with well-behaved boundary, $F(x)$ is a function with positive values, $\nabla$ denotes the gradient and $|$.$| represents the Euclidean norm. The right-$ hand side $F(x)$ is typically supplied as known input. Physically, the solution $\mathrm{u}(\mathrm{x})$ is the shortest time needed to travel from the boundary $\partial \Omega$ to $x$ inside $\Omega$, with $F(x)$ being the time cost (not speed) at $x$.

In the special case when $\mathrm{F}=1$, the solution represents the signed distance from $\partial \Omega$.

\section{A. Variational form of Eikonal equation.}

The variational formulation of Eikonal equation

$$
\sqrt{(\nabla \varphi(x))^{T} M(x) \nabla \varphi(x)}=1 \quad x \in \Omega
$$

describes a traveling wave through the domain $\Omega$ with given heterogeneous, anisotropic velocity information $M$. The solution $\varphi(x)$ denotes the time when the wave arrives at point $x$.

The computational domain for solving the Eikonal equation is discretized by planar-sided tetrahedra whose vertices are the discretization points storing the discrete solution. The continuous solution is represented by a linear interpolating within each tetrahedron.

\section{B. Definition of the local solver.}

The local solver calculates the solution at a vertex based on given solution of the Eikonal equation on three other vertices of a given tetrahedron.

The solution $\phi(x)$ or the travel time for each vertex is computed using the linear approximation on its one-ring tetrahedra (all the neighboring tetrahedra of vertex $x$ ). This is also the bulk of the work computed by the local solver.

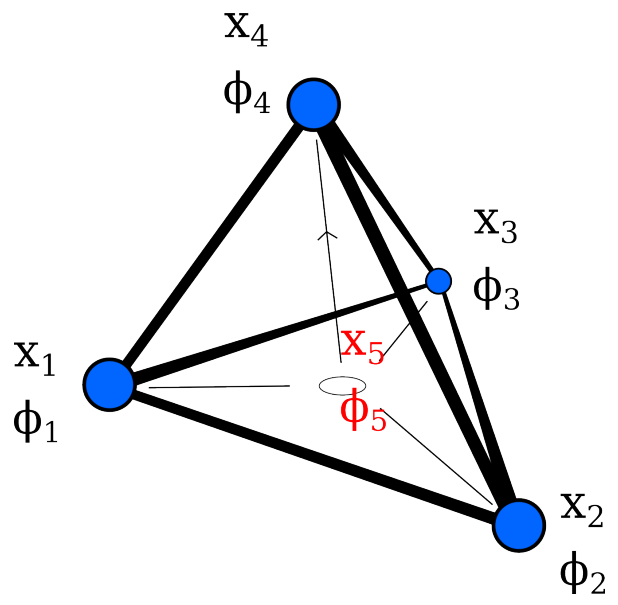

Fig. 2. The local solver updates the value of the approximation at the vertex $x_{4}$ from the values $\varphi_{1}, \varphi_{2}$ and $\varphi_{3}$ of the other three vertices. The wave propagating towards $x_{4}$ leaves the triangle $\triangle 1,2,3$ at $x_{5}$.

We use an upwind scheme to compute the solution $\phi_{4}$, assuming the values $\phi_{1}, \phi_{2}$, and $\phi_{3}$ comply with the causality property of the Eikonal solutions [6]. Since the speed function is constant within each tetrahedron then the travel time to $x_{4}$ is determined by the time associated with the segment from $x_{5}$ to $x_{4}$ as depicted in Figure 2, that minimizes the solution value at to $x_{4}$. For this reason we first compute the coordinates of $x_{5}$ which is the point the wave-front intersects the plane defined by the vertices $x_{1}$, $x_{2}$ and $x_{3}$. Then we check whether $x_{5}$ is contained in that plane.

Denoting $\phi_{5,4}=\phi_{4}-\phi_{5}=\sqrt{e_{5,4}^{T} M e_{5,4}}$ as travel time from $x_{5}$ to $x_{4}$, a minimization problem regarding $\phi_{4}$ can be formulated. Based on Fermat's principle [7] the goal is to find the location of $x_{5}$ that minimizes $\phi_{4}$. For achieving this we use the mathematical description as explained in [3]. If $x_{5}$ does not fall within the plane formed by the three other vertices, then we apply the $2 \mathrm{D}$ local solver used in [4] to the faces $\triangle_{1,2,4}, \triangle_{1,3,4}$, and $\triangle_{2,3,4}$ and select the minimal solution from among the three. The same applies also for the surface solutions which means that 
in case the minimal solutions falls outside the tetrahedral face, then we consider the solutions along the edges.

With the growth of smart devices or embedded systems, low-memory-footprint programs have regained importance once more. Low-memory-footprint programs are of paramount to applications on embedded platforms where memory is often constrained to within a few MBs so much so that developers typically sacrifice efficiency (processing speeds) just to make program footprints small enough to fit into the available RAM. Because one of our goals is the transfer of the local solver to an Android device we have been very careful during the building process of our solver in order to keep the memory footprint as low as possible. Of course this is not the only reason since also in the case of parallel architectures with limited high-bandwidth memory, the memory footprint of the local solver becomes a bottleneck to performance.

\section{FAst Iterative Method (FIM)}

FIM was initially proposed in [5] as a new iterative method to solve the Eikonal equation in parallel architectures, especially on GPUs. The idea that FIM is particularly fitted for parallel computing was reinforced in [8] where the authors adapted FIM in their work to build a fine-grained parallel model that works great on large scale applications. Our work is also another proof of this. The proposed method uses a modification of the active list update scheme as presented in [5], [4], [3] combined with the local solver described above designed for unstructured tetrahedral meshes with inhomogeneous anisotropic speed functions. We adopted this method in our work and modified the algorithm in such way that during the expansion phase less computations are done.

The method solves the Eikonal equation selectively on the mesh, by maintaining a narrow band, forming the wave front, called the active list. This list is used to store all the nodes that are currently being updated. The algorithm iteratively updates the active list nodes until they converge. During each iteration the active list nodes are updated and the list is modified in such way that new nodes, whose current solution values are downwind(neighbors with larger value) w.r.t the updated converged node, are added and the converged (consistent solution with their neighbors) nodes are removed. Thus, a vertex is removed from the active list when its solution is up to date with respect to its neighbors, and a vertex is appended to the list when the value of any potentially upwind neighbor has changed. Convergence of the algorithm to a valid approximation of the Eikonal equation was proven in [5]. The algorithm continues looping through the active list until the list is empty. This description followed the paper by Jeong and Whitaker [5].

The modification of the algorithm by us is twofold.
First, the active list initialization method in [5], [4] goes through all the vertices of the domain and checks if any neighboring vertex is source then adds it to the list. Instead of going through all the vertices of the domain our algorithm goes through all the source vertices, finds their neighboring vertices and adds them to the list.

Second, a very important condition is not reflected to the algorithm in [5], [4] where all the neighboring are processed during the expansion phase. Instead we process only the downwind neighbors. The larger the valences of mesh the larger the performance improvement since without this condition more unnecessary computations for solving also upwind neighbors are done. This change improves the performance by $20 \%$ approximately tested for TBunny_C mesh with maximum valence of 30 .

\section{IMPLEMENTATION ON SERIAL AND MULTI THREADED CPUS.}

In this section we discuss how the proposed algorithm can be efficiently mapped to multi threaded CPU's. The multithreaded version of the algorithm is straightforward since the update of the active list nodes do not depend on one another. For this reason the multi threaded version of

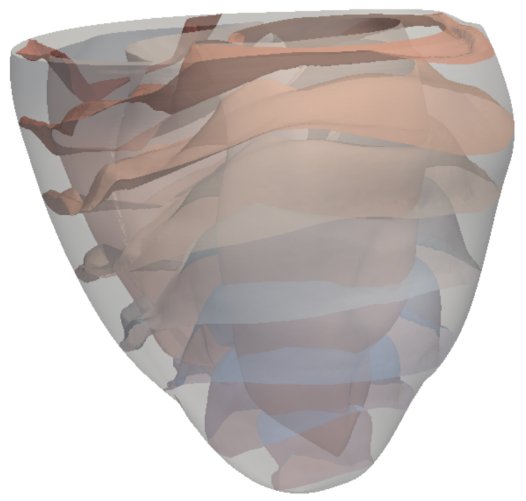

Fig. 3. Arrival time $\varphi(x)$ ranging from 0 (bottom) to 1 (top).

the algorithm can be derived very simply by just partitioning the active list for each iteration and assign the work of each sublist to one thread. We just make sure to have a dynamic and even partitioning which allows an even workload among threads and avoids unnecessary splitting and reassembling preparations on the active list during each iteration which can be very costly by impairing the performance. Each thread is updating its own active sublist but the solution is synchronized against the solution vector $\mathrm{U}$ where all the values for each node are kept.

The shared memory parallelization on CPU has been implemented using OpenMP. We improved the algorithm, implemented it on various hardware with very good results 
and also transferred the solver for a coarse model to a tablet computer and other hand-held devices for potential clinical use.

Due to the splitting of the wave front, the parallel version results in a slightly different convergence history and in minor differences in the solutions. This happens only in the parallel version. In the iterative algorithm we have always the same results and the same convergence history. It is true that the updates are independent from one another and wouldn't affect the result if they are executed in different order. This is the main reason why it is possible to map the iterative algorithm easily to the parallel one. But what changes on different executions is the number of available nodes of the basis, which are the nodes with solution value different from infinity, that are used to find a solution in one node. This because the update of a node depends only on the available neighboring nodes as explained in section II-B. It may happen that in the first execution one of the values is infinity then based on the algorithm we calculate the 2D solution and the 1D solution. This because a 3D solution is not possible for the moment. Since the algorithm is iterative, it updates the nodes until they converge. So in the next iteration, when the same node is solved again, this infinity node becomes available and a 3D solution is found. It is possible that the best solution value is found on the 2D case previously calculated and not in the 3D case. Yet, in the next execution it may happen that all the three nodes of the basis are available from the beginning and since we found a solution on the 3D case we do not search for the solution on the 2D case which might be a better solution. So in the first execution for the same nodes a better solution is found than in the second execution. This can be avoided by changing the algorithm and instead calculating all the possible solution values, $1 \mathrm{D}, 2 \mathrm{D}$ and $3 \mathrm{D}$ and get the minimal value but this would require more computational time. Anyway this would result in better solution values and a unique convergence history. This phenomena does not happen in the iterative algorithm since all the nodes have always the same available nodes for computing their solutions. On the other hand, in parallel, other nodes that were not available in the iterative version might have been solved already giving in this way more options to one node to find a better solution.

The parallel algorithms have been tested on workstations and on Android devices. We have a very short convergence time of the algorithm and good quality results, see Figure 3 wherein the wave propagation looks very smooth.

\section{CUDA IMPLEMENTATION.}

When designing an algorithm that will be used in a streaming unit such as a GPU, it is very important to try to optimize for throughput and not for latency. Because
GPU's are built to optimize for throughput and not for latency. Having this in mind but also the concept of coalesced access pattern we designed our algorithm based on one critical point: allowing data redundancy in order to achieve good coalesced memory access, throughput and occupancy. The main idea of the Eikonal update scheme is to find the solution of one node calculating for all the one-ring tetrahedra. It means that in the general case one thread is supposed to solve for one node by doing the computations and solving for all its neighboring tetrahedra. Unfortunately, we would have non-coalesced access pattern, smaller number of threads (low level of parallelism), low throughput and a poor occupancy. A better solution consists in calculating a priori all the neighboring tetrahedra indices for each node in the active list, store them in an array in global memory and then assign each element (tetrahedra) to one thread. Of course the information will be redundant as shown in Figure 5 on the last array, but we loose in memory to gain in performance . In this way we achieve a better coalesced memory access, more threads to run (increased parallelism) and a better bandwidth which are the three most important concepts for a fast GPU algorithm.

\section{A. Using SCAN for Compaction.}

In order to successfully apply this idea we use the parallel SUM SCAN algorithm and its most important applications such as stream compaction and address generation for efficiently scattering the results into memory as presented in [9].

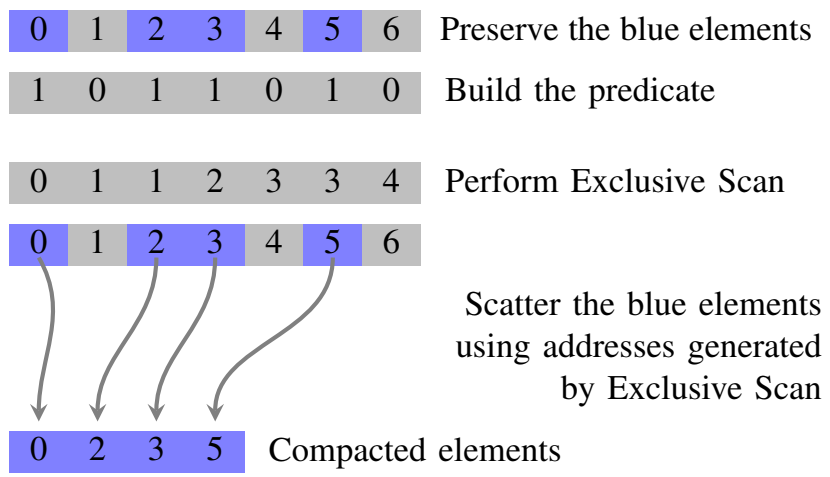

Fig. 4. Address Generation and Compaction process.

Because we do not use any specific parallel data structure for managing the active list in our CUDA implementation we use an old fashion (C, Fortran) array with ones and zeros in order to distinguish between nodes in the active list and nodes not in the active list as you can notice on the InitializeActiveList procedure in Algorithm 1 line 8 and 12. This list now can be easily managed by some atomic update operations. In this way we are able to add, remove 
and also update the active list. But when the problem comes to solving for each active element then we will end up with a branchy code where most of the threads will remain idle. This would happen because we would solve only for the active elements and the threads processing non-active (zero) elements are going to remain idle. This is very bad for the performance. For this reason we use compaction. Stream compaction requires two steps, a scan and a scatter as shown in Figure 4. By using scan on the predicate array we build another array of accumulated sum excluding the last element (Algorithm 1, line 18). That is why it is called exclusive SUM SCAN. The result values are nothing else but the generated scatter addresses and if you look only the blue elements they are just the exact addresses which will be used for the scatter step. Based on these addresses the active elements are scattered to the compacted array, last array of Figure 4. Compaction is useful when there is a large number of elements to compact and the computation on each surviving element is expensive. This is exactly our case and it resulted to be one of the kernels with a very good performance in our implementation.

\section{B. Using SCAN for Data Management.}

Figure 5 presents the rest of the process until solving all the nodes in the active list.

\section{$\begin{array}{lllll}0 & 2 & 3 & 5 & \text { Compacted active list indices (1) }\end{array}$}

\section{$\begin{array}{lllll}2 & 3 & 4 & 1 & \text { Total neighbors for each index (2) }\end{array}$}

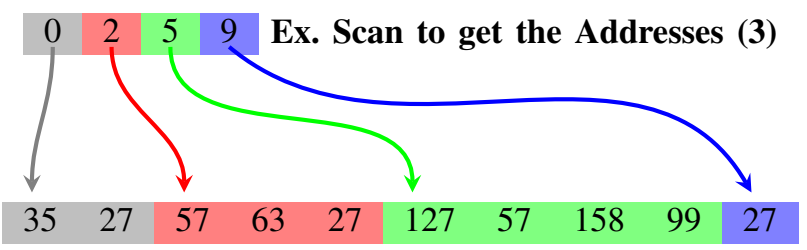

Neighbor Tetrahedra Indices (4)

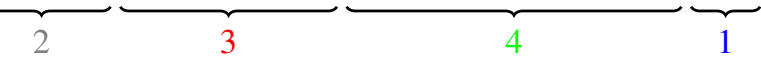

Fig. 5. Data management for achieving a high computational density on the GPU.

The first array in the figure denotes the compacted active list generated by using the process from Figure 4. Based on the compacted active list, we compute the total number of neighboring tetrahedra for each node of the active list (Algorithm 1, line 20) and store them to a temporary array as in step 2 in Figure 5. Then the scan algorithm is applied to this temporary array to generate the addresses which will be used to scatter the neighboring tetrahedra indices to the last result array (Algorithm 1, line 21-22).

For example, from the Figure 5 the first two elements are copied starting at address 0 , the next 3 elements starting at address 2 and so on. The information on the last array is redundant. This scheme allows us to achieve coalesced access and also to increase the parallelism by increasing the working number of threads. One thread per element is used for solving the elements into the last array as in Algorithm 1, line 23-27. The algorithm uses the same scheme later on when solves for all the neighboring nodes of the converged active list nodes.

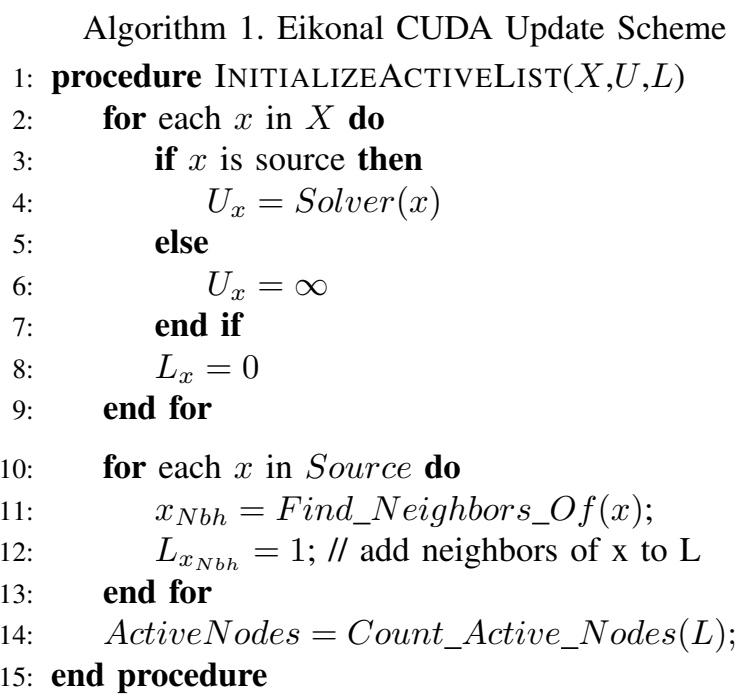

16: procedure UPDATEPOINTSINL $(X, U, L)$

17: $\quad$ while ActiveNodes $>0$ do

18: $\quad s A d d r=$ Exclusive_SCAN $(\mathrm{L})$; // calculate scatter address from $\mathrm{L}$.

19:

20: $c L i s t=$ Compact_Active_List $(s A d d r, L)$; $n b h N r=$ Count_Nbhs(cList); // count the total number of neighboring // tetrahedra for each $x$ in $L$ $s A d=$ Exclusive_SCAN $(n b h N r)$; // calculate addresses from $n b h N r$ elemList = Scatter_elements $(s A d, c L i s t)$; // find all the neighboring tetrahedra // for each $x$ in $L$ and store them // based on the address generated in 'line : 21 ' for each elem in elemList do $p=U_{x}$; $q=\operatorname{Solver}(x$, elem $)$ // where $\mathrm{x}$ is the reference vertex of the // element elem (the vertex to be solved)

predicate $_{x}=(|p-q|<$ $d_{e}$ psilon $\left.*(1+(|p|+|q|) / 2)\right)$; end for $s=$ Exclusive_SCAN(predicate); 
conList $=$ Compact_Predicate $(s$, predicate $)$; // generate the converged active list.

30: $n b h N r=$ Count_Nbhs(conList); $/ /$ count the total number of neighboring $/ /$ points for each converged $x$ in conList

31: $\quad L_{x}=0 ; / /$ Remove $x$ from $L$

32: $\quad s A d=$ Exclusive_SCAN $(n b h N r)$;

// calculate addresses from $n b h N r$

33: $\quad$ ptList $=$ Scatter_elements $(s A d$, conList $)$;

// find all the neighboring Points for each

$/ /$ converged $x$ in $L$ and store them based on // the address generated in 'line : 32'

34:

47: end procedure

\section{TRANSFER OF THE SOLVER TO AN ANDROID TABLET COMPUTER.}

Today complex medical diagnostic devices are becoming more portable, robots are becoming higher functioning, drones are learning to navigate autonomously. As a result, the computational requirements for such devices are increasing exponentially, while their size, weight and power limits continue to decrease. NVIDIA Shield Tablet is one of the android tablet devices which incorporates one of the most innovative mobile processor to feature a CUDA-capable GPU such as NVIDIA Tegra K1 192 core Kepler GPU 2.2 GHz ARM Cortex A15 CPU with 2 GB RAM. Tegra K1 is a tiny but full-featured mobile processor designed for development of embedded and mobile applications which supports the complete CUDA Toolkit version 6.0. To implement and test our CUDA algorithm to such device we used NVIDIA CodeWorks for Android [10]. These devices are evolving with the time and this was one reason more to continue our work on the CUDA implementation for such mobile processors. Actually we have two different working solvers on Android devices. The first targeting the general Android devices which uses the OpenMP version of the solver and the other targeting those devices who incorporate cudacapable GPUs which is using the CUDA version of the solver. Of course there are a lot of issues we have been facing during implementation and one of these is the lack of possibility to debug and profile the CUDA code on Android. This makes it very difficult to find the issues and slows down the progress. But also with the current tools it is more than possible to host the HPC practice into such devices with satisfactory results.

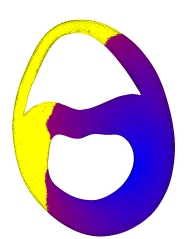

(a) Wave traveling through the domain.

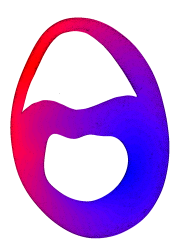

(b) Wave reached the end point in the domain.
Fig. 6. Traveling wave through a slice of heart domain in two different states simulated using OpenGL ES 2.0.

We use OpenGL ES 2.0 [11] for rendering the 3D graphics on the Android device. By using it we achieve to simulate the wave propagation on a heart mesh. In the future we plan to use OpenGL ES 2.0 to realize an interactive change of material properties on the tablet and their direct influence on the simulation. As shown in the Figure 6 we achieved to transfer the solver and to display the traveling wave based on the solution from the Eikonal Equation. But of course there is still much work to do in this direction. Actually we have applied the same algorithm we used for CUDA in the previous section to the Android CUDA version. But we believe that more research work need to be done in order to optimize the algorithm for these tiny computers which hosts a much less powerful device than a normal GPU but with a great future. Different processing power of the GPU influences a lot on the way the algorithm is built. So the next try will be adapting Algorithm 1 for these very promising mobile processors such as Tegra K1. Anyway, with what we have implemented so far we get a factor of 8 speedup compared to the OpenMP implementation tested on NVIDIA shield tablet as shown in Table I. 
TABLE I. Comparison between CUDA and OpenMP implementations on Shield Tablet (Android).

\begin{tabular}{|c|c|c|c|}
\hline Meshes & \# Tetrahedras & $\begin{array}{r}\text { CUDA } \\
\text { sec. }\end{array}$ & $\begin{array}{r}\text { OpenMP } \\
\text { sec. }\end{array}$ \\
\hline slab(st & 240,000 & 1.84 & 8.59 \\
\hline TBunnyC2 & 266,846 & 3.78 & 29.56 \\
\hline TBunnyC & $3,073,529$ & 30.26 & 257.14 \\
\hline
\end{tabular}

It is very important to emphasize that the OpenMP version runs on the Shield CPU: ARMv7 quad core $2.2 \mathrm{Ghz}$, which means only four threads are used to solve the equation. As conclusion, the GPU version on mobile devices gives us far better results then the OpenMP implementation on ARM processors.

\section{NUMERICAL TESTS AND COMPARISONS.}

We present the results for the numerical tests performed on various machines with different processing power and we use the desktop processor Intel Core i7-4700MQ CPU @ $2.40 \mathrm{GHz}$ as baseline. It took the sequential code version with the iterative implementation of the base algorithm from [5] 60.86 seconds by using one core. Taking advantage of all 4 cores plus the hyperthreading reduced the run time to 17.41 seconds.

Table II presents results for two meshes performed on various CPUs with different processing power. We achieved the best computational time of 10.62 seconds on the Intel Xeon CPU E5-2660 2.20 GHz with 10 cores plus hyperthreading. In this case the list is divided into 20 sublists and then processed asynchronously by each thread.

TABLE II. CPU shared memory runtime in sec.

\begin{tabular}{lrrrrr}
\hline \multirow{4}{*}{ Meshes } & & Intel & Intel & Intel & Intel \\
& \# Tets & Core i7 & Xeon & Xeon & \multicolumn{1}{c}{ Xeon } \\
& $2.40 \mathrm{GHz}$ & $2.00 \mathrm{GHz}$ & $2.67 \mathrm{GHz}$ & $2.20 \mathrm{GHz}$ \\
& $\times 4+\mathrm{HT}$ & $\times 16$ & $\times 12$ & $\times 10+\mathrm{HT}$ \\
\hline TBunnyC & $3,073,529$ & 17.41 & 13.35 & 13.14 & $\mathbf{1 0 . 6 1}$ \\
TBunnyC2 & 266,846 & 1.38 & 1.31 & 1.16 & 1.20 \\
\hline
\end{tabular}

The results on the table for TBunnyC mesh are intentionally sorted in the descending order. The second row results for the smaller mesh, TBunnyC2, does not behave in the same way. If we take a closer look at the two last columns we observe that for smaller meshes the clock speed has a greater impact than for bigger meshes. It proves ones more that the performance improvements are no longer driven primarily by increased clock speeds, but by parallelism. To better understand how well the parallel strategies are working, we present some scaling results from tests performed on both our TBunnyC and TBunnyC 2 meshes and on Intel Xeon $2.67 \mathrm{GHz}$ CPU as shown in Table III. The number of threads varies from 1 to 12 which is the maximum number of processing cores on the machine we tested our implementation. From the results one can see that our parallel algorithm scales very well.

TABLE III. Scaling results on Intel Xeon 2.67Ghz.

\begin{tabular}{crr}
\hline \# Threads & $\begin{array}{r}\text { TBunnyC2 } \\
\text { sec. }\end{array}$ & $\begin{array}{r}\text { TBunnyC } \\
\text { sec. }\end{array}$ \\
\hline 1 & 4.47 & 65.67 \\
\hline 2 & 2.74 & 38.75 \\
\hline 4 & 1.75 & 23.13 \\
\hline 6 & 1.43 & 18.53 \\
\hline 8 & 1.22 & 15.71 \\
\hline 10 & 1.18 & 14.43 \\
\hline 12 & 1.16 & 13.14 \\
\hline & &
\end{tabular}

It would be interesting to make a performance comparison with the existing approaches such as in [3]. But because of different meshes we are testing against, it is difficult to make a fair comparison. The closest we can get is when we compare both of ours different heart meshes:

Mesh 3 in [3]: a heart model with 437,355 vertices and $2,306,717$ tetrahedra whose maximum valence is 68 .

TBunnyC: a heart model with 547,680 vertices and $3,073,529$ tetrahedra whose maximum valence is 30 (Figure 1.).

Velocity is the same for both: a heterogeneous anisotropic correlated random symmetric positive-definite speed tensor.

Regarding the CPU we tested against Intel i7 920 which was the only one available in our lab which could be fairly compared to the Intel i7 965 extreme used in [3].

The table below shows the convergence time in both cases. It seems that we are faster compared to the result in [3] although the different valences have an impact on the run time.

TABLE IV. Performance comparison with other approaches.

\begin{tabular}{lccc}
\hline Meshes & CPU & \# Threads & $\begin{array}{c}\text { Convergence } \\
\text { time in sec. }\end{array}$ \\
\hline TBunnyC & Intel i7 $920 @ 2.67 \mathrm{GHz}$ & 4 & $\mathbf{2 1}$ \\
Mesh 3 & Intel i7 965 extreme @ 3.2GHz & 4 & $\mathbf{5 6}$ \\
\hline
\end{tabular}

When designing the update scheme for the CUDA parallel algorithm we focused on the goal to improve the OpenMP version by at least a factor of 4 . We achieved a factor much better than that for the Android device, see Table I but in comparison to the ARM processor.

Table $\mathrm{V}$ shows the fair comparison between a consumer GPU and a good CPU. Our first CUDA version, implemented using NVIDIA CUDA API [12], without the domain decomposition achieves an acceleration larger 
than 2. We expect another factor of 2 once the domain decomposition ideas are realized in the CUDA code. Notice also the behavior of the algorithm for smaller meshes. The OpenMP implementation is a lot faster for the slab mesh but the CUDA algorithm performs much better for larger meshes. This proves the fact that GPUs optimize for throughput and not for latency as done the CPUs.

TABLE V. Comparison between CUDA and OpenMP (GTX 970 vs. Intel i7-4700MQ @2.40GHz ×4 +HT).

\begin{tabular}{lrrr}
\hline Meshes & \# Tetrahedras & $\begin{array}{r}\text { CUDA } \\
\text { sec. }\end{array}$ & $\begin{array}{r}\text { OpenMP } \\
\text { sec. }\end{array}$ \\
\hline slab(structured) & 240,000 & 0.657 & 0.354 \\
TBunnyC2 & 266,846 & 0.913 & 1.673 \\
TBunnyC & $3,073,529$ & 8.347 & 18.0039 \\
\hline
\end{tabular}

We want to mention also that based on Table $\mathrm{V}$ and Table I results one can make a fair comparison between two different GPUs, in our case the GEFORCE GTX 970 and the Tegra K1. From the results we notice that we have only a factor of 4 difference in favor of GTX 970 of course. This yields to the conclusion that transferring the solver to such android devices is very promising.

\section{CONCLUSIONS AND FUTURE WORK.}

In this paper, we have presented the FIM for solving the inhomogeneous anisotropic Eikonal equation over fully unstructured tetrahedral meshes. We introduce our algorithms for CPU (iterative), shared memory parallelization and CUDA. Solving the equation iteratively for single CPU gives very good results but solving on parallel gives better results on solution values since more solutions are found on Tetrahedrons than on Triangle surface sides or edges of the Tetrahedrons. The execution time also improves a lot as showed in the previous sections. We describe the detail data structures and algorithms for streaming architectures and how does the careful management of data allows a higher computational density on the GPU which yields then a satisfactory speed up factor compared to the OpenMP implementation. Then we propose to transfer the Eikonal Solver to an Android Tablet in order to understand that it is possible to host a HPC program on such devices and also with satisfactory results especially when these devices incorporate a CUDA-capable GPU for development of embedded and mobile applications such as Tegra K1. These kind of devices are becoming more and more useful for medical use which is another reason to transfer the application to an Android Device.

As a future work, we will focus on employing the domain decomposition to our CUDA parallel algorithm which we are currently implementing. From our analysis so far and preliminary results we conclude that this version will increase the utilization of the shared memory, decrease the non-coalesced access level and significantly increase of computational density. Similar work has been done for a fully parallelized solution of the wave equations as in [13] by employing multiple GPUs for large scale applications to avoid the limited global memory in one GPU. However our management strategy of employing the available blocks to run the active sub-domains and the implementation of 16-bit Floating Point Data [14] will allow to run the domain decomposition version also in one GPU and with better results on multiple accelerator cards and cluster computing. To give the users a better graphical experience we will realize an interactive change of material properties on the tablet computer and their direct influence on the simulation. But of course solving on the tablet will not be the only way. We plan to combine grid/cloud computing for the Eikonal equations with post processing on the tablet computer.

\section{ACKNOWLEDGEMENT}

The authors gratefully acknowledge support from Erasmus Mundus JoinEUsee PENTA scholarship and from NAWI Graz.

\section{REFERENCES}

[1] N. F. Otani, "Computer modeling in cardiac electrophysiology." $J$. Comput. Phys., pp. 21-34, 161 (2010).

[2] T. J. Barth and J. A. Sethian, "Numerical schemes for the HamiltonJacobi and level set equations on triangulated domains." J. Comput. Phys., pp. 1-40, 145 (1998).

[3] Z. Fu, R. M. Kirby, and R. T. Whitaker, "Fast iterative method for solving the eikonal equation on tetrahedral domains." SIAM J. Sci. Comput., vol. 35, no. 5, pp. C473-C494, 2013.

[4] Z. Fu, W.-K. Jeong, Y. Pan, R. M. Kirby, and R. T. Whitaker, "A fast iterative method for solving the eikonal equation on triangulated surfaces." SIAM J. Sci. Comput., vol. 33, pp. 24682488, 2011.

[5] W.-K. Jeong and R. T. Whitaker, "A fast iterative method for eikonal equations.” SIAM J. Sci. Comput., vol. 30, pp. 2512-2534, 2008.

[6] J. Qian, Y.-T. Zhang, and H.-K. Zhao, "Fast sweeping methods for eikonal equations on triangulated meshes." SIAM J. Numer. Anal., pp. 83-107, 45 (2007).

[7] D. D. Holm, "Geometric mechanics: Part I: Dynamics and symmetry, 2nd ed." Imperial College London Press, London, UK, 2011.

[8] F. Dang and N. Emad, "Fast iterative method in solving eikonal equations : a multi-level parallel approach." Procedia Computer Science, vol. 29, pp. 1859-1869, 2014.

[9] M. Harris, "Parallel prefix sum (scan) with cuda." http:// www.eecs.umich.edu/courses/eecs570/hw/parprefix.pdf, 2007.

[10] NVIDIA, "NVIDIA CodeWorks for Android." https://developer.nvidia.com/codeworks-android.

[11] Android, "OpenGL ES API," https://developer.android.com/guide/topics/graphics/opengl.html.

[12] NVIDIA, "CUDA C programming guide." http://docs.nvidia.com/cuda/cuda-c-programming-guide.

[13] M. Noack, "A two-scale method using a list of active sub-domains for a fully parallelized solution of wave equations." Journal of Computational Science, vol. 11, pp. 91-101, 2015.

[14] NVIDIA, "New Features in CUDA 7.5." https:// devblogs.nvidia.com/parallelforall/new-features-cuda-7-5. 